\title{
Role of hydrogen in hydrogen-induced layer exfoliation of germanium
}

\author{
J. M. Zahler, ${ }^{1}$ A. Fontcuberta i Morral, ${ }^{1,2}$ M. J. Griggs, ${ }^{1}$ Harry A. Atwater, ${ }^{1}$ and Y. J. Chabal ${ }^{3}$ \\ ${ }^{1}$ Thomas J. Watson Laboratory of Applied Physics, California Institute of Technology, Pasadena, California 91125, USA \\ ${ }^{2}$ Walter Schottky Institut, Technische Universität München, 85748 Garching, Germany \\ ${ }^{3}$ Laboratory for Surface Modification, Rutgers University, Piscataway, New Jersey 08854, USA
}

(Received 3 August 2006; revised manuscript received 12 November 2006; published 9 January 2007)

\begin{abstract}
The role of hydrogen in the exfoliation of Ge is studied using cross-sectional transmission electron microscopy, atomic force microscopy, and multiple-internal transmission mode Fourier-transform infrared absorption spectroscopy and compared with the mechanism in silicon. A qualitative model for the physical and chemical action of hydrogen in the exfoliation of these materials is presented, in which H-implantation creates damage states that store hydrogen and create nucleation sites for the formation of micro-cracks. These micro-cracks are chemically stabilized by hydrogen passivation, and upon annealing serve as collection points for molecular hydrogen. Upon further heating, the molecular hydrogen trapped in these cracks exerts pressure on the internal surfaces causing the cracks to extend and coalesce. When this process occurs in the presence of a handle substrate that provides rigidity to the thin film, the coalescence of these cracks leads to cooperative thin film exfoliation. In addition to clarifying the mechanism of $\mathrm{H}$-induced exfoliation of single-crystal thin Ge films, the vibrational study helps to identify the states of hydrogen in heavily damaged Ge. Such information has practical importance for the optimization of $\mathrm{H}$-induced layer transfer as a technological tool for materials integration with these materials systems.
\end{abstract}

DOI: 10.1103/PhysRevB.75.035309

PACS number(s): 68.55.Ln, 78.30.-j, 68.37.Lp, 68.35.Ja

\section{INTRODUCTION}

Hydrogen-induced exfoliation of semiconductors combined with wafer bonding techniques has been applied for more than a decade to the layer transfer of thin films onto nonlattice matched substrates. H-implantation is a technological process that allows the insertion of a hydrogenated layer in a material. $\mathrm{H}^{+}$ions are accelerated with relatively high voltages ( $80 \mathrm{keV}$ in our case) and are stopped at a certain distance from the surface, producing damage in the material mainly in the form of vacancies in the matrix. For energetic reasons, the created damage states tend to store hydrogen and create nucleation sites for the formation of micro-cracks. These micro-cracks are believed to be chemically stabilized by hydrogen passivation, and upon annealing serve as collection points for molecular hydrogen. Upon further heating, the molecular hydrogen trapped in these cracks exerts pressure on the internal surfaces causing the cracks to extend and coalesce. When this process occurs in the presence of a handle substrate that provides rigidity to the thin film, the coalescence of these cracks leads to exfoliation. This process was first developed for silicon ${ }^{1}$ and is the basis of the industrial production of silicon-on-insulator (SOI) substrates. Wafer bonding and layer transfer has now been applied to semiconductors other than silicon such as Ge, InP, GaAs, and GaN. ${ }^{2-6}$ The mechanism of H-induced exfoliation has been extensively studied in the case of silicon, ${ }^{7,8}$ and, more recently, in the case of InP. ${ }^{9}$ The early work of Weldon et al. provided important conclusions: (i) implantation induced defects serve to trap $\mathrm{H}$ within the Si substrate. (ii) A broad distribution of vacancy hydrogen defect structures is the main reservoir for hydrogen that contribute to the exfoliation process. For subcritical doses of $\mathrm{H}$, the spectral fingerprint for these vacancies is significantly diminished. (iii) Agglomerated $\mathrm{VH}_{3,4}$ defect structures are precursors to the formation of extended internal surfaces that serve to accumulate $\mathrm{H}_{2}$ and build internal pressure. Furthermore, the (100) and (111) platelet structures observed in TEM serve as nucleation points for the agglomeration of vacancy-hydrogen defect structures; (iv) The concentration of bound hydrogen is reduced upon annealing, as indicated by a decrease in the integrated intensity of the Si-H modes. Yet, the majority of hydrogen was shown by forward recoil scattering (FRS) to remain in the Si rather than to diffuse out of the semiconductor. Thus, the loss of bound hydrogen leads to the formation of molecular $\mathrm{H}_{2}$ confined to internal structures. (v) $\mathrm{H}_{2}$ trapped in internal surfaces was shown by mass spectroscopy to have the physical role of pressurizing the micro-cracks to provide the energy necessary for their extension through the material. (vi) By studying bonded implanted samples along with free surfaces, it was shown that the presence of the handle substrate plays only a dynamic role as a stiffener during the internal pressurization of the micro-cracks. This induces lateral crack growth, but shows no spectroscopic difference from the free surface sample.

The $\mathrm{H}$-induced exfoliation mechanism of $\mathrm{Ge}$ is expected to be qualitatively similar to this process summarized above for Si. However, the chemical activity of $\mathrm{Ge}$ and $\mathrm{Si}$ is often quantitatively different. Although the characteristics of isolated $\mathrm{H}$-stabilized defects are similar in $\mathrm{Si}$ and $\mathrm{Ge},{ }^{10,11}$ it is important to investigate the behavior of high concentration of hydrogen in Ge during the exfoliation process. Such a study is not only important to understand the fundamentals of $\mathrm{H}$-induced layer exfoliation of germanium, but also as a source of reference of the $\mathrm{Ge}-\mathrm{H}$ related vibrational modes in heavily implanted germanium.

This paper is structured as follows. In Sec. II the experimental details are given. Cross-sectional transmission electron microscopy (XTEM) and multiple internal transmissionmode Fourier-transform infrared absorption spectroscopy (MIT-FTIR) measurements are then presented in Sec. III. 
These results are discussed in Sec. IV to arrive at a model for the role of hydrogen in the H-induced layer exfoliation of $\mathrm{Ge}$ with conclusions presented in Sec. V.

\section{EXPERIMENTAL DETAILS}

Double-side polished Ge(100) arsenic-doped wafers were used for this study. The resistivity was above $10 \Omega \mathrm{cm}^{-1}$, in order to minimize free-carrier absorption during infrared absorption measurements. The substrates were implanted with $80 \mathrm{keV} \mathrm{H}^{+}$at an incidence angle of $7^{\circ}$ at three doses: 2 $\times 10^{16}, 5 \times 10^{16}$, and $1 \times 10^{17} \mathrm{~cm}^{-2}$. The wafer temperature was kept at or below room temperature as measured with a thermocouple in contact with the back surface of the substrate by cooling the back-side of the implant stage during implantation, thereby minimizing the dynamic annealing of the substrates during the implantation process. After implantation, the wafers were cut into pieces for the realization of cross-sectional transmission electron microscopy (XTEM), atomic force microscopy (AFM), and multiple internal transmission Fourier transform infrared spectroscopy (MIT-FTIR) measurements. The $40 \times 15 \times 0.5 \mathrm{~mm}^{3}$ MIT samples were beveled at $45^{\circ}$ along the short sides, allowing radiation to enter normal to the bevel edge, and totally internally reflect on the Ge faces (the $45^{\circ}$ internal incidence angle is much larger than the $14^{\circ}$ critical angle for the Ge/air interface) a total of 70 times (35 times on each side). All spectra were collected with a Thermo-Nicolet spectrometer using a liquidnitrogen cooled mercury cadmium telluride detector with a low frequency cutoff near $600 \mathrm{~cm}^{-1}$. All implanted Ge pieces were annealed isochronally in a nitrogen ambient. Spectra were acquired following approximately $15 \mathrm{~min}$ isochronal annealing steps of 59,131,170,221,297, 339, 399, and $501{ }^{\circ} \mathrm{C}$ in order to follow the evolution of the chemical state of hydrogen. The temperature of the sample during anneal steps was monitored with a Sensarray thermocoupleinstrumented wafer, accurate to $\pm 0.1{ }^{\circ} \mathrm{C}$.

\section{RESULTS}

\section{A. TEM and AFM measurements}

For the understanding of the process of $\mathrm{H}$-induced layer exfoliation, the chemistry of hydrogen with Ge determined by FTIR is a key. Additionally, by transmission electron microscopy measurements it is possible to determine the density and identity of the crystallographic defects present in the crystal after ion implantation and how the defects evolve during annealing.

In Fig. 1 XTEM micrographs show both low- and highmagnification images of an as-implanted Ge sample implanted on a gas-cooled stage with $\mathrm{H}^{+}$at $80 \mathrm{keV}$ to a total dose of $1 \times 10^{17} \mathrm{~cm}^{-2}$. In Fig. 1(a), the broad distribution of the implant induced damage immediately following implant corresponds to the brighter zone, at about $600 \mathrm{~nm}$ from the front surface. The position and thickness matches well with the predicted distribution of $\mathrm{H}$ within the Ge substrate. In Fig. 1(b), a higher magnification XTEM image of the implanted region shows the presence of a large concentration of platelet defect structures parallel to the (100) planes and a a)

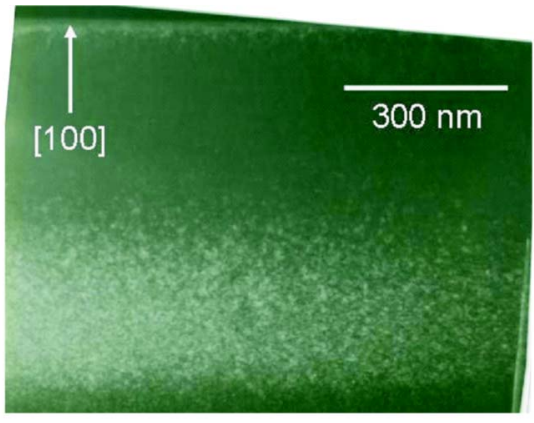

b)

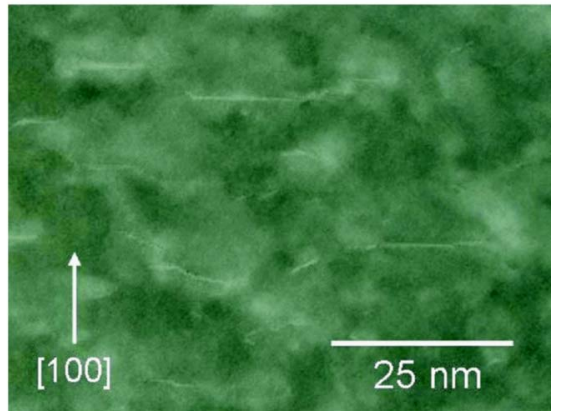

FIG. 1. (Color online) (a) An XTEM image of Ge as-implanted with $80 \mathrm{keV} \mathrm{H}^{+}$to a dose of $1 \times 10^{17} \mathrm{~cm}^{-2}$ on a gas cooled stage revealing the broad damage distribution structure of Ge implanted under these conditions. (b) A high-magnification image of the heavily damaged region near the peak range of the implant showing a large concentration of (100) platelet defect structures and a smaller concentration of (111) platelets.

smaller concentration of platelets inclined $25^{\circ}$ with respect to the (100), parallel to the (111) planes. In the following, the platelets oriented parallel to (100) and (111) planes will be named (100) and (111) platelets. In order to discern the effect of implantation from the effect of dynamic/static annealing on the nano-structure of the Ge, X-TEM measurements were realized on implanted $\mathrm{Ge}$ on an un-cooled stage before and after annealing at $250{ }^{\circ} \mathrm{C}$ for $10 \mathrm{~min}$. The results are illustrated in Fig. 2 by low- and high-magnification. The implant dose and energy correspond exactly to those of Fig. 1. While the dynamic temperature during implantation is higher for a sample implanted on a cooled stage than it is for an uncooled sample, the subsequent anneal of $250{ }^{\circ} \mathrm{C}$ is thought to be considerably higher than the peak temperature reached during implantation - as on the contrary exfoliation could occur during implantation. As a consequence, the images in Fig. 2 are assumed to be typical of the evolution of the defect structure in $\mathrm{H}^{+}$-implanted Ge. The defect structure and distribution is shown in the low-magnification image in Fig. 2(a). The initial stages of the formation of micro-cracks at the predicted peak range of $560 \mathrm{~nm}$ are observed. These cracks are referred to as micro-cracks in reference to the distribution of crack lengths from 25 to $100 \mathrm{~nm}$. Figure 2(b) shows the micro-cracks at increased magnification. Further inspection shows an increase in the number of (111) and (100) platelets following the $250{ }^{\circ} \mathrm{C}$ anneal. Finally, Fig. 2(c) captures the process by which the micro-cracks coalesce by jumping between (100) micro-cracks at differing depths. This process is consistent with the physical model of (100) platelets opening 
a)

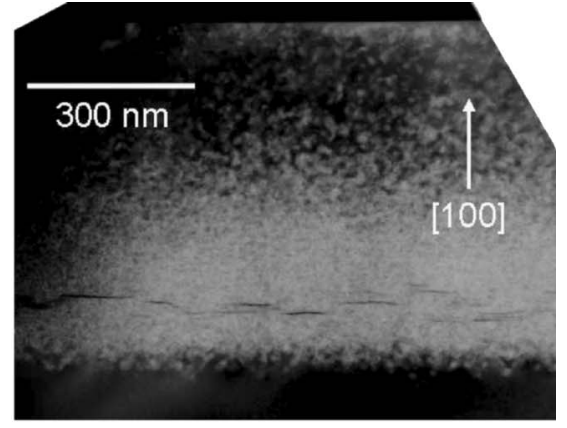

b)

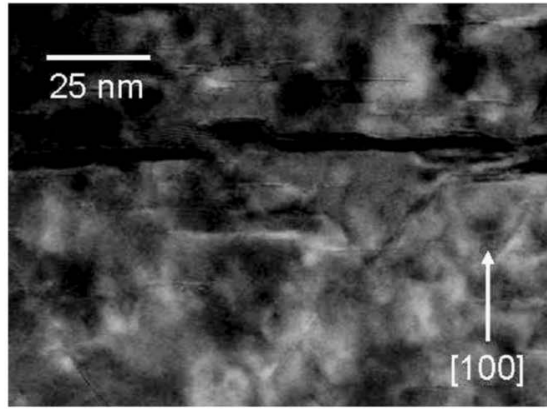

c)

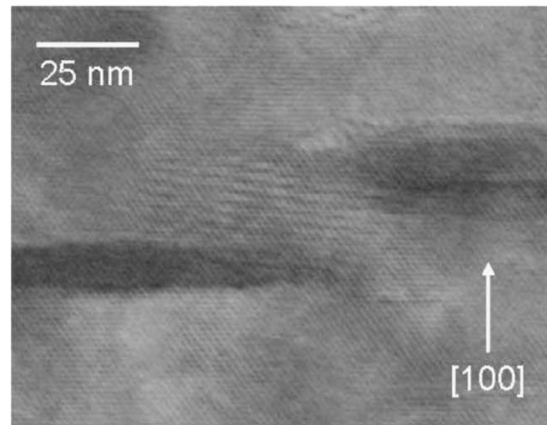

FIG. 2. (a) An XTEM image of Ge implanted with $80 \mathrm{keV} \mathrm{H}^{+}$to a dose of $1 \times 10^{17} \mathrm{~cm}^{-2}$ without active cooling following an anneal to $250{ }^{\circ} \mathrm{C}$ for $10 \mathrm{~min}$. (b) A high magnification XTEM image of the micro-crack region under the same conditions presence of (100) and (111) hydrogen platelets and micro-cracks just beginning to form in the material. (c) A high-resolution XTEM image of Ge implanted with $80 \mathrm{keV} \mathrm{H}^{+}$to a dose of $1 \times 10^{17} \mathrm{~cm}^{-2}$ without active cooling following an anneal to $250{ }^{\circ} \mathrm{C}$ for 10 min under close magnification showing the coalescence of nanocracks into microcracks that eventually lead to exfoliation.

at various implantation depths to form micro-cracks. These micro-cracks are distributed in depth in the film around the range of the implant. To complete the exfoliation process, the micro-cracks ripen to form extended macro-crack structures by the joining of micro-cracks at various depths in the $\mathrm{H}$-implanted region of the Ge substrate through crackjumping along planes that otherwise would not preferentially form a cleavage plane.

To corroborate the evolution of the structural properties of the implanted Ge annealed at higher temperatures, we present some AFM measurements of the surface. While the wafer surface topology is unchanged following $\mathrm{H}^{+}$implantation, the presence of blisters at the surface can be observed by AFM once the micro-cracks start coalescing and open cavities formed. Figure 3(a) shows the development of blis-
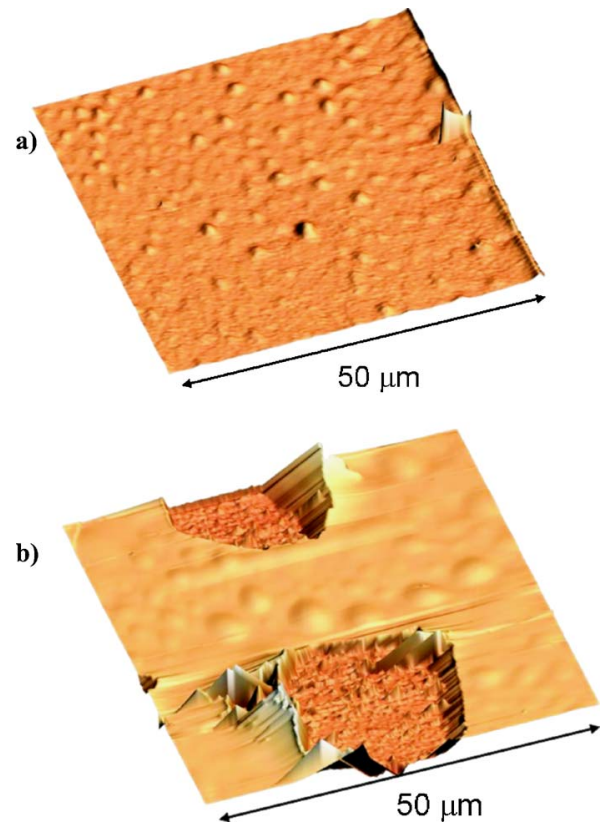

FIG. 3. (Color online) (a) A contact mode AFM image of an $\mathrm{H}$-implanted $\mathrm{Ge}$ surface following an anneal to $250^{\circ} \mathrm{C}$ for $10 \mathrm{~min}$ for an $80 \mathrm{keV} \mathrm{H} \mathrm{H}^{+}$dose of $1 \times 10^{17} \mathrm{~cm}^{-2}$ without active cooling. (b) A contact mode AFM image of the same surface following an anneal to $300{ }^{\circ} \mathrm{C}$ for $10 \mathrm{~min}$.

ters on the surface of an implanted Ge substrate annealed for $10 \mathrm{~min}$ at $250{ }^{\circ} \mathrm{C}$. There is a dense coverage of small surface protrusions corresponding to blisters formed in the early stages of the exfoliation process. Following an additional 10 minute anneal at $300{ }^{\circ} \mathrm{C}$, an AFM image shown in Fig. $3(\mathrm{~b})$, it is shown that the disperse blisters have ripened into larger blisters. The ripening of the blisters provides a direct support for the physical picture suggested by the XTEM images. That is, the exfoliation process is initiated with the formation of a dense network of micro-scale cracks around the peak range of the $\mathrm{H}$-implant. Upon further annealing, as seen by the crater-like structure found in Fig. 3(b), the rupture of the blister is observed, most probably due to the accumulation and further increase of the internal gas pressure of the $\mathrm{H}_{2}$ inside the blister.

XTEM and AFM analysis of $\mathrm{H}^{+}$-implanted Ge have shown that implantation creates some extended defects such as platelets. These platelets become more defined and grow during annealing before the rupture of the internal cavities and exfoliation of a surface layer of the wafer. MIT-FTIR measurements presented in the next section serve to elucidate the chemical role of hydrogen in the exfoliation of $\mathrm{H}^{+}$ implanted Ge.

\section{B. MIT-FTIR spectroscopy measurements}

\section{Basic principles}

TEM and AFM are valuable for showing the physical phenomena that occur during the exfoliation and layer transfer of a Ge thin film in H-implantation-induced exfoliation but are not sensitive to chemical changes in the process. In 
contrast, vibrational spectroscopy is best-suited to understand the chemical state and role of $\mathrm{H}$ in the exfoliation process, which dominate the microscopic mechanisms. Specifically, MIT-FTIR spectroscopy, performed as a function of the electric field polarization, can provide information on the orientation of Ge-H modes and/or the location of bound hydrogen. Indeed, the intensity of $\mathrm{Ge}-\mathrm{H}$ modes polarized normal to the surface (or crack direction) can be greatly enhanced if the $\mathrm{Ge}-\mathrm{H}$ species are located in a region of low index of refraction. ${ }^{7,12}$ Following a simple three-layer model, the absorption of radiation upon transmission at an interface region is

$$
\frac{\Delta I}{I_{0}}=\frac{2 \pi d}{\lambda} \cdot \frac{1}{n \cdot \cos \Theta}\left[\cos ^{2} \Theta \cdot \operatorname{Im} \tilde{\boldsymbol{\epsilon}}+n^{4} \sin ^{2} \Theta \cdot \operatorname{Im} \frac{-1}{\tilde{\boldsymbol{\epsilon}}}\right],
$$

where $\Theta$ is the internal angle of incidence, $d$ and $\tilde{\epsilon}$ are the effective interface thickness and dielectric function, respectively, and $n$ is the substrate index of refraction (germanium in our case). The absorption has two different contributions, the first proportional to the imaginary part of epsilon associated with absorption parallel to the interface and a second proportional to the inverse of epsilon, associated with absorption perpendicular to the interface. The ratio between the absorption of parallel and perpendicular radiation is then

$$
\frac{\perp}{\|}=\frac{n^{4} \sin ^{2} \Theta}{|\widetilde{\epsilon}|^{2} \cos ^{2} a} .
$$

For an incidence angle of $45^{\circ}$, the enhancement of one of the components comes from the relatively much lower index of refraction of the interfacial region. This situation occurs for hydrogen at the surface of cracks only when the fracture is well developed (i.e., when the index is close to 1) and the absolute enhancement greatly depends on the distance of the crack to the outer surface. However, in cases where the cracks are in a distance within the wavelength from the outer surface of the sample, it is also necessary to take into account the interference of the incident and reflected beam. Under these conditions, the intensity of each polarization is also proportional to the square of the electrical field components which is given by the expressions, ${ }^{9}$

$$
\begin{gathered}
\left|\mathbf{E}_{s}\right|^{2}=4 E_{0}^{2} \cdot\left|\sin \Theta \cos \left(k_{z} \cdot+\frac{1}{2} \Delta \Psi\right)\right|^{2}, \\
\left|\mathbf{E}_{p}\right|^{2}=E_{0}^{2} \cdot\left|\sin \Theta \cos \left(k_{z} \cdot z+\frac{1}{2} \Delta \Psi\right)\right|^{2} \\
+E_{0}^{2} \cdot\left|\cos \Theta \cos \left(k_{z} \cdot z+\frac{1}{2} \Psi_{p}\right)\right|^{2}, \\
\frac{1}{2} \Psi_{p}=-\arctan \frac{n^{2} \sqrt{n^{2} \sin ^{2} \Theta-1}}{n \cdot \cos \Theta},
\end{gathered}
$$

$$
\frac{1}{2} \Delta \Psi=\frac{\pi}{2}-\frac{1}{2} \Psi_{p},
$$

where $z$ is the distance of the low index region from the surface (600 nm in our case) and $\mathbf{E}$ is the electrical field, $\mathbf{k}$ the wave vector and the subindexes correspond to the different components. The comparison of spectra taken in $s$ - and $p$-polarization provides the additional information necessary to distinguish the configuration and location of hydrogen at various steps of the process. From Eqs. (1)-(3) the formation of a low index layer (the cracks) would lead to an increase of absorption of the modes taken with $p$-polarization in comparison to that of $s$-polarized spectra. This enhancement does not require a large gap, as was demonstrated in the case of the bonding of two H-terminated Si wafers, where the distance between two Si surfaces is believed to be $\approx 4 \AA .^{13}$ The most important parameter is the perfection of the lateral extend of the crack, since the IR beam is affected by the effective index of the crack. The location of neighboring cracks should be within the average crack length to insure that there is a definable average crack position with a lower index of refraction.

\section{Total implantation dose dependence}

The three implantation doses studied exhibit three different physical behaviors: (1) $2 \times 10^{16} \mathrm{~cm}^{-2}$-a sub-critical dose that does not blister upon annealing; (2) $5 \times 10^{16} \mathrm{~cm}^{-2}$-an intermediate dose that exhibits blistering only at temperatures above $500{ }^{\circ} \mathrm{C}$; and, (3) $1 \times 10^{17} \mathrm{~cm}^{-2}$ - a high dose that leads to lower-temperature blistering. MIT-FTIR spectra of the three different implanted Ge wafers maintained at room temperature (i.e., no anneals after implantation) are compared in Fig. 4 for measurements performed both under $s$ - and $p$-polarization. The spectra taken with $s$-polarization show much richer spectral details, because none of the bands are strongly enhanced. In contrast, the spectra taken with $p$-polarization are dominated by the enhancement of features associated with $\mathrm{H}$ located in the low density area of the material (cracks and internal surfaces), making it difficult to distinguish $\mathrm{H}$ located in discrete defects in the bulk (i.e., outside of the lower-index, heavily-damaged region). For this reason, the assignment of discrete defect modes at low temperature is done using spectra taken with $s$-polarization. Important additional information to positively identify discrete defect modes is obtained from temperature dependence measurements (Figs. 5-7), from which the thermal stability of the defects can be derived. Table I catalogs the dominant peaks observed in the different implant conditions as a function of temperature. The peaks present in spectra measured with both with $s$ - and $p$-polarizations are indicated in parentheses. Additionally, Table I lists values reported in the literature for well-studied defect structures of hydrogen on Ge surfaces, in interstitial and vacancy defects in Ge, which is helpful to interpret the spectra and to follow the evolution of discrete and extended defects measured by MIT-FTIR in H-implanted germanium.

\section{Temperature dependence}

The temperature-dependence of all $\mathrm{H}$-induced modes is shown for three different doses: $2 \times 10^{16} \mathrm{~cm}^{-2}$ in Fig. 5, 5 


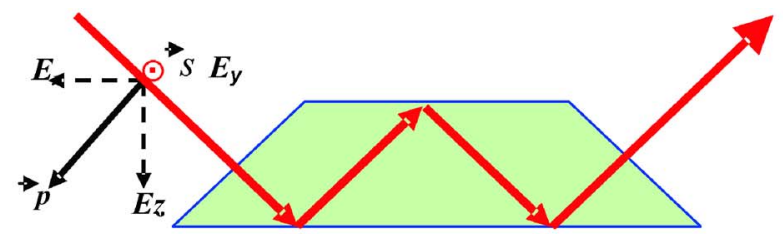

b)

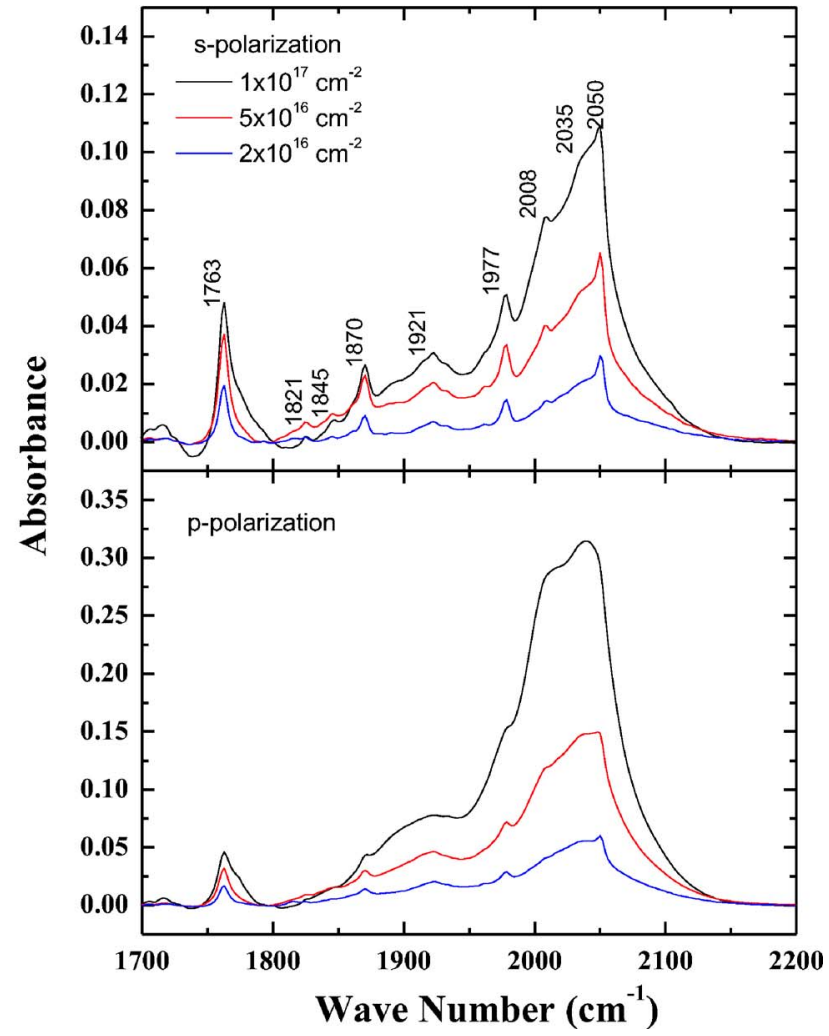

FIG. 4. (Color online) (Color online) MIT-FTIR of unannealed $\mathrm{H}$-implanted samples of $2 \times 10^{16} \mathrm{~cm}^{-2}, 5 \times 10^{16} \mathrm{~cm}^{-2}$, and 1 $\times 10^{17} \mathrm{~cm}^{-2}$ showing the discrete $\mathrm{Ge}-\mathrm{H}$ defect modes seen in this study. The top panel shows spectra taken with $s$-polarization and the bottom panel shows spectra taken with $p$-polarization.

$\times 10^{16} \mathrm{~cm}^{-2}$ in Fig. 6, and $1 \times 10^{17} \mathrm{~cm}^{-2}$ in Fig. 7. Examining the spectra taken with $s$-polarization, it appears the temperature dependence of the peaks attributed to discrete defects is consistent for all three implant conditions. For instance, features located at 1763 and $1979 \mathrm{~cm}^{-1}$ have been associated with the $\mathrm{Ge}-\mathrm{H}_{2}^{*}$ defect structure. The position of these features, measured at $333 \mathrm{~K}$, is close to the values reported by Nielsen et al. measured at $10 \mathrm{~K}$ for the $\mathrm{Ge}-\mathrm{H}_{2}^{*}$ anti-bonding $\left(1763 \mathrm{~cm}^{-1}\right)$ and bond centered $\left(1989 \mathrm{~cm}^{-1}\right)$ stretch modes, respectively. ${ }^{14}$ Although there is a small discrepancy for the bond centered stretch, both modes display the same thermal evolution reported by Nielsen (e.g., they vanish after a $443 \mathrm{~K}$ anneal, which compares favorably to $438 \mathrm{~K}$ reported by Nielsen et al.). Additional support for this assignment is the observation of a feature with the same temperature dependence at $763 \mathrm{~cm}^{-1}$ very close to the frequency of $765 \mathrm{~cm}^{-1}$ reported for the $\mathrm{Ge}-\mathrm{H}_{2}^{*}$ bending mode. ${ }^{15}$ The small differences with the values reported by Nielsen $e t$ $a l$. are most likely due to the measurement temperature.

In addition to the frequencies assigned to the $\mathrm{H}_{2}^{*}$ stretch modes, there are at least seven other modes in the range from
$1700-2200 \mathrm{~cm}^{-1}$. Among them, the feature at $1870 \mathrm{~cm}^{-1}$ is near the frequency of the stretch mode of the dihydrogenated self-interstitial, $\mathrm{IH}_{2}$, at $1880 \mathrm{~cm}^{-1}$ reported by Nielsen et al. The temperature behavior of this defect also corroborates this assignment with the feature disappearing by $221{ }^{\circ} \mathrm{C}$, slightly below the literature reported value of $240{ }^{\circ} \mathrm{C} .{ }^{14}$ The strong feature located at $2050 \mathrm{~cm}^{-1}$ is near the reported value of the $\mathrm{VH}_{4}$ defect at $2061.5 \mathrm{~cm}^{-1}$, but also close to the stretching frequency of the germanium trihydride $\mathrm{GeH}_{3}$. This feature is seen to vanish between anneals of 297 and $339^{\circ} \mathrm{C}$, very close to the report by Nielsen et al. in which the feature attributed to the $\mathrm{VH}_{4}$ defect was seen to vanish at $317^{\circ} \mathrm{C}$. As it will be discussed later, this feature constitutes a prominent contribution appearing after annealing that may be correlated to the formation of internal surfaces and subsequent micro-cracks.

The mode at $2008 \mathrm{~cm}^{-1}$ observed with $s$-polarization is present for all implantation doses at room temperature and remains stable up to $\approx 365{ }^{\circ} \mathrm{C}$ (it disappears abruptly between 339 and $399^{\circ} \mathrm{C}$ ). The attribution of this mode is more controversial. Its thermal dependence and the fact that it does not exhibit any enhancement when measuring the absorption under $p$-polarization suggest that it can be assigned to a discrete defect structure that contributes molecular hydrogen to the exfoliation process and might form a precursor mode. As the mono-vacancy $\mathrm{VH}_{2}$ has already been attributed to the peak present at $1979 \mathrm{~cm}^{-1}$, one possible assignment for this peak would be the $\mathrm{V}_{2} \mathrm{H}_{6}$ defect with a mode reported at $2014.9 \mathrm{~cm}^{-1}, 8$ which has been shown to be unstable above $347^{\circ} \mathrm{C}$. The $\approx 7 \mathrm{~cm}^{-1}$ discrepancy could be due to strain in the Ge itself or to interaction between $\mathrm{H}$ in opposite side of the vacancy, which would be expected for larger vacancies $\left(\mathrm{V}_{n} \mathrm{H}_{n+4}, n>2\right)$. Since the dihydride mode on $\mathrm{Ge}(100)$ is at $2020 \mathrm{~cm}^{-1}$, it is likely that the spectral region between 1015 and $2020 \mathrm{~cm}^{-1}$ encompasses modes of larger vacancies. Interaction between opposite surfaces of such large vacancies does lead to a red shift. For instance, in the case dihydride on $\mathrm{Ge}(100)$ surfaces, a shift towards lower wavenumbers occurs when the hydrogen is located in internal hydrogen terminated surfaces, due to both the interaction between the close hydrogen atoms and opposite surfaces. ${ }^{16,17}$ This assignment of this mode would also explain its enhancement in spectra measured with $p$-polarization, and its red shift during annealing. The remaining weak modes observed in the $1700-2200 \mathrm{~cm}^{-1}$ frequency range at room temperature cannot be assigned at present, but are assumed to correspond to hydrogen-decorated interstitials and vacancies induced during the implantation of the Ge substrate. Additionally, there is a broad background in this region similar to (although not as intense as) a broad feature observed in Si and assigned to a distribution of multi-vacancy defects of the form $\mathrm{V}_{x} \mathrm{H}_{y}$ and hydrogenated self-interstitials of the form $\mathrm{I}_{x} \mathrm{H}_{y}{ }^{1}$ A possible explanation for the difference between the background intensity in the present study and the Si-H study is that Ge would suffer less lattice damage following implant due to the larger mass of the $\mathrm{Ge}$ atom.

Figures 5(a) and 5(b) show the evolution of the spectra taken in both $s$ - and $p$-polarizations, respectively, as a function of isochronal anneal temperature for a sub-critical dose of $2 \times 10^{16} \mathrm{~cm}^{-2}$. The spectra in Fig. 5(a) best show the 


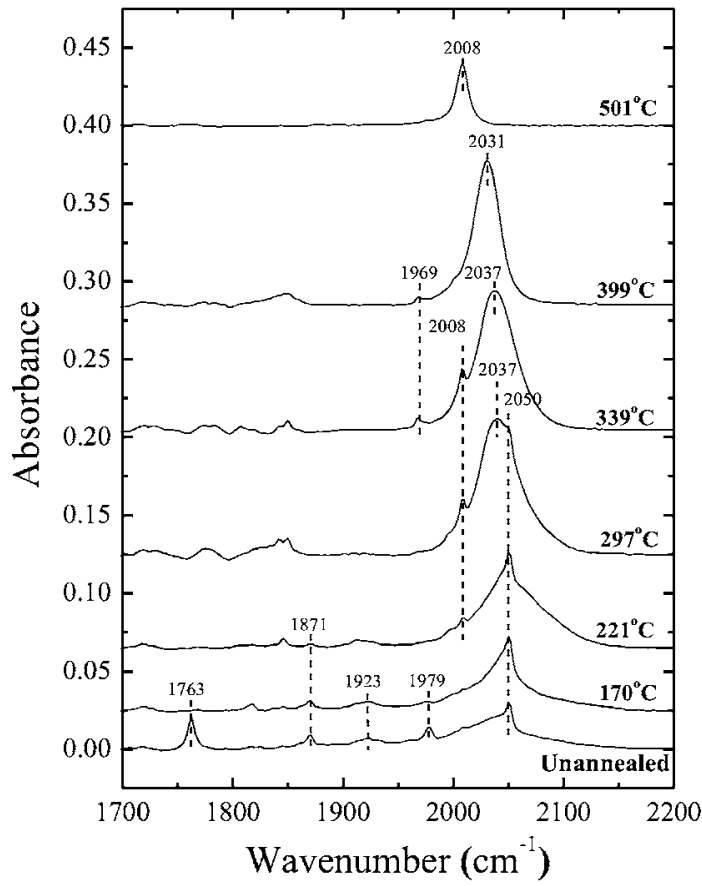

(a)

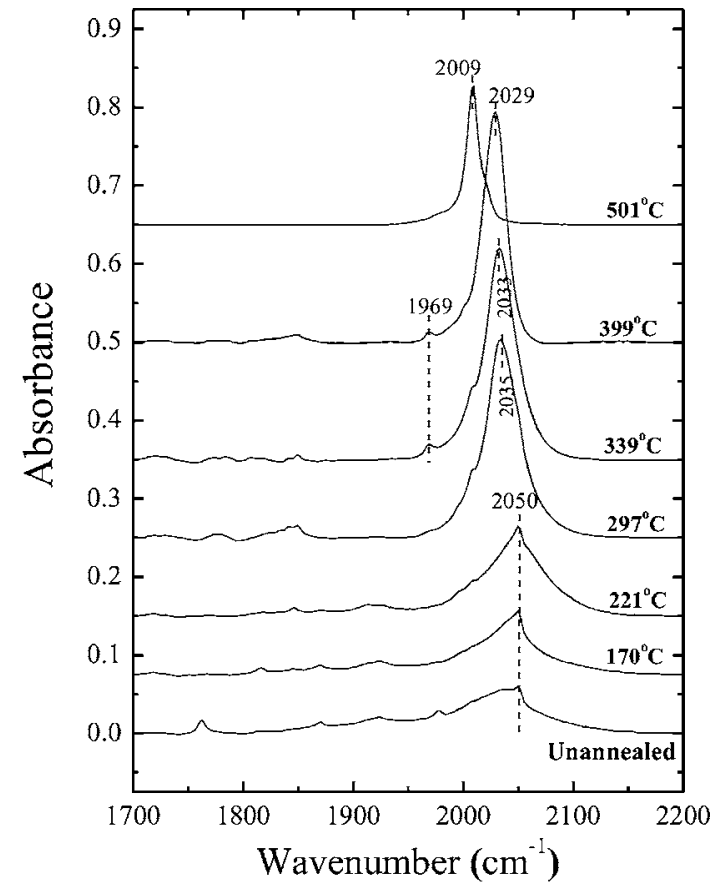

(b)

FIG. 5. (a) MIT-FTIR spectra taken with $s$-polarization for $2 \times 10^{16} \mathrm{~cm}^{-2}$ implanted Ge as a function of the isochronal annealing temperature. (b) MIT-FTIR spectra taken with $p$-polarization for $2 \times 10^{16} \mathrm{~cm}^{-2}$ implanted $\mathrm{Ge}$ as a function of the isochronal annealing temperature.

modes associated with the dominant discrete defect modes removed by relatively low temperature anneals. At temperatures above $170{ }^{\circ} \mathrm{C}$ the dominant modes are all located in the $1950-2100 \mathrm{~cm}^{-1}$ range, which encompasses the vacancyhydrogen defect modes as well as extended surfaces. Upon annealing, the features in the $1950-2100 \mathrm{~cm}^{-1}$ range sharpen and evolve, with the disappearance (or red shift) of the $2050 \mathrm{~cm}^{-1}$ mode into a single peak with greatly reduced integrated intensity at $2008 \mathrm{~cm}^{-1}$.

The weak features seen in the $1700-1900 \mathrm{~cm}^{-1}$ range after annealing at 297,339 , and $399^{\circ} \mathrm{C}$ are difficult to assign unambiguously because they overlap partially with contamination features observed after implantation and also after annealing. ${ }^{18}$ After subtracting strong bands attributed to contamination in this region, it is not reliable to attempt to assign the residual peaks to any $\mathrm{Ge}-\mathrm{H}$ structure, particularly when none have been reported in the literature.

The spectra in Fig. 5(b) taken with $p$-polarization show the evolution of the bands that are clearly enhanced. As was previously noted, these enhanced bands dominate the spectrum and make it difficult to study $\mathrm{Ge}-\mathrm{H}$ modes that are not enhanced (e.g., residing outside of the internal cracks or with a polarization parallel to the surface). In Fig. 6(b) the spectrum taken with -polarization for the unannealed sample shows minimal enhancement of the $2050 \mathrm{~cm}^{-1}$ mode tentatively attributed to the $\mathrm{VH}_{4}$ defect. After annealing to $221{ }^{\circ} \mathrm{C}$ the $2050 \mathrm{~cm}^{-1}$ band is strengthened and broadened. However, at $297{ }^{\circ} \mathrm{C}$ a strong mode at $2035 \mathrm{~cm}^{-1}$ develops at the expense of the $2050 \mathrm{~cm}^{-1}$ mode. This indicates that the low refractive index region of the implanted $\mathrm{Ge}$ is becoming better defined and that the feature being enhanced is a major constituent of that region with a strong $z$-axis dipole. For anneals of 339 and $399{ }^{\circ} \mathrm{C}$ the enhanced feature shifts downward, and finally at $501{ }^{\circ} \mathrm{C}$ the dominant feature is greatly reduced in intensity and is found at $2009 \mathrm{~cm}^{-1}$. This spectral evolution is in good agreement with results previously obtained by Stein et al. for hydrogen implanted Ge in the absence of internal voids in the Ge. ${ }^{19}$

The evolution of $\mathrm{Ge}-\mathrm{H}$ stretch modes discussed above is associated with germanium implanted with a dose under the threshold of exfoliation. We are going now to analyze a germanium implanted at the threshold dose $\left(5 \times 10^{16} \mathrm{~cm}^{-2}\right)$. The use of a dose close to the exfoliation threshold allows the observation of the exfoliation in a more gradual way. The spectra in Figs. 6(a) and 6(b) show the corresponding evolution of the spectra taken with $s$ - and $p$-polarization, respectively. As with the $2 \times 10^{16} \mathrm{~cm}^{-2}$ sample, the mode at $2050 \mathrm{~cm}^{-1}$ dominates the spectrum up to $221^{\circ} \mathrm{C}$. However, in contrast to the case of $2 \times 10^{16} \mathrm{~cm}^{-2}$ implantation dose, there is a strong band in the unannealed spectrum at $2008 \mathrm{~cm}^{-1}$ attributed to the dihydride present at extended internal surfaces that continues to grow through $339^{\circ} \mathrm{C}$. Following an annealing at $399^{\circ} \mathrm{C}$ new features are observed. The dominant spectral feature is now located at $2025 \mathrm{~cm}^{-1}$, but there are several modes at lower frequencies, including modes at 2000, 1990, and $1969 \mathrm{~cm}^{-1}$. The slight shoulder at $1990 \mathrm{~cm}^{-1}$ increases in intensity to become the dominant feature at $501{ }^{\circ} \mathrm{C}$. This is once again shifted by $10 \mathrm{~cm}^{-1}$ from the asymmetric stretch of the $\mathrm{Ge}(100)$ monohydride terminated surface reported by Chabal, but the shift can again be attributed to the fact that hydrogen is located in 


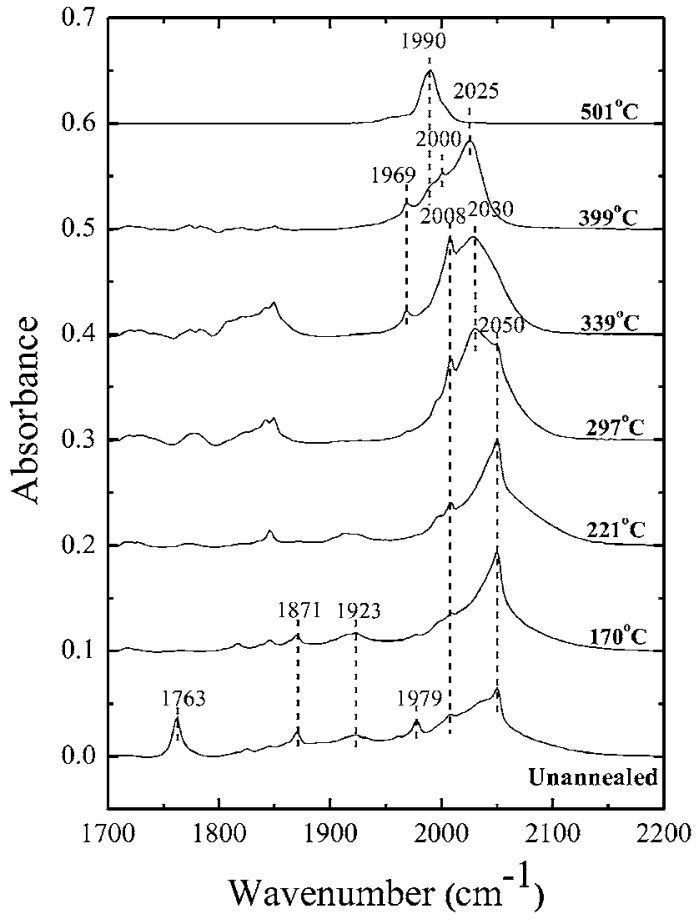

(a)

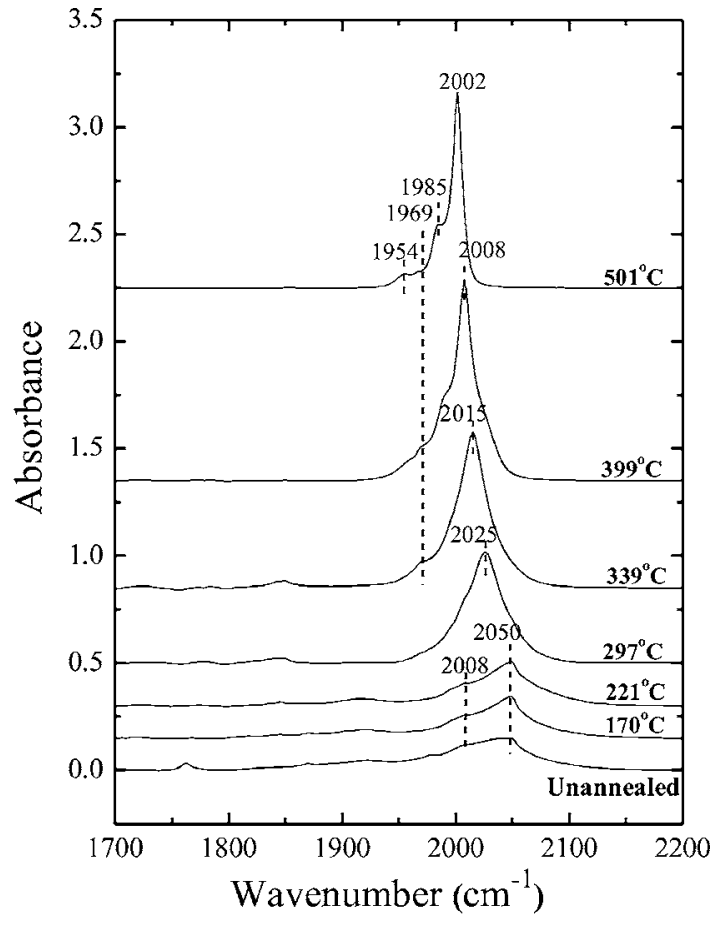

(b)

FIG. 6. (a) MIT-FTIR spectra taken with s-polarization for $5 \times 10^{16} \mathrm{~cm}^{-2}$ implanted Ge as a function of the isochronal annealing temperature. (b) MIT-FTIR spectra taken with $p$-polarization for $5 \times 10^{16} \mathrm{~cm}^{-2}$ implanted Ge as a function of the isochronal annealing temperature.

internal and not open surfaces. ${ }^{20}$ Following the $501{ }^{\circ} \mathrm{C}$ anneal there remains a slight shoulder extending to $\approx 1950 \mathrm{~cm}^{-1}$. This shoulder encompasses the reported values of $1978 \mathrm{~cm}^{-1}$ for the Ge(111) surface mono-hydride. ${ }^{9}$ Thus, these data provide evidence of the presence of (100) and (111) internal surfaces at $501{ }^{\circ} \mathrm{C}$.

In Fig. 6(b), the spectra taken with $p$-polarization, critical steps in the evolution of the $\mathrm{Ge}-\mathrm{H}$ structure necessary for exfoliation are again observed by the increase in the enhancement. The main characteristics of these measurements are the relatively simple nature of the spectra (only one or two peaks) and relatively little enhancement from the asimplanted condition through a $221{ }^{\circ} \mathrm{C}$ anneal. Upon annealing to $297{ }^{\circ} \mathrm{C}$ there is an onset of enhancement with a strong peak at $2025 \mathrm{~cm}^{-1}$. Following the $339^{\circ} \mathrm{C}$ anneal, the enhanced peak continues to shift downward to $2015 \mathrm{~cm}^{-1}$. The sample annealed to $399^{\circ} \mathrm{C}$ shows a further shift in the dominant peak down to $2008 \mathrm{~cm}^{-1}$ accompanied by increased spectral detail in the low-frequency shoulder of this peak, which at this temperature appears to have contributions from modes at 1969 and $2000 \mathrm{~cm}^{-1}$. Both of these features were also observed in the spectrum taken with $s$-polarization, suggesting that they are modes with a parallel dipole. Finally, upon annealing to $500{ }^{\circ} \mathrm{C}$, a temperature at which the onset of blistering is observed but at which exfoliation has not yet occurred, the enhanced peak shifts further to $2002 \mathrm{~cm}^{-1}$ and now has clearly distinguishable shoulder features at lower frequencies due to peaks estimated to be located at 1954 , 1969 , and $1985 \mathrm{~cm}^{-1}$. The symmetric stretch mode of the $\mathrm{Ge}(100)$ surface monohydride being reported in a much lower frequency of $1991 \mathrm{~cm}^{-1}$, we attribute the strong feature shifting from 2025 to $2002 \mathrm{~cm}^{-1}$ to the evolution of isolated trihydrides on $\mathrm{Ge}(100)$ surfaces to the accumulation forming germanium dihydrides..$^{21,22}$ The shoulder at $1985 \mathrm{~cm}^{-1}$ is near the Ge(111) surface monohydride value seen by Stein et al. of $1978 \mathrm{~cm}^{-1}$. Furthermore, it is consistent with the physical picture of the ripening of micro-cracks into an extended crack structure by fractures along the higher index planes such as the (111) separating the micro-cracks that lie primarily on the $\mathrm{Ge}(100)$ plane.

The MIT-FTIR spectra for the low-temperature-blistering condition $1 \times 10^{17} \mathrm{~cm}^{-2}$ taken with both $s$ - and $p$-polarizations as a function of temperature are shown in Figs. 7(a) and 7(b), respectively. The spectrum taken with $s$-polarization for the unannealed sample shows the dominant discrete features that were previously noted. As with the 2 and $5 \times 10^{16} \mathrm{~cm}^{-2}$ samples, the mode at $2050 \mathrm{~cm}^{-1}$ dominates the spectrum up to $221{ }^{\circ} \mathrm{C}$. However, in contrast to the case of lower implantation doses, there is a strong band in the unannealed spectrum at $2008 \mathrm{~cm}^{-1}$ attributed to germanium dihydrides in extended internal surfaces, that continues to grow through $339{ }^{\circ} \mathrm{C}$. At $297{ }^{\circ} \mathrm{C}$ a strong peak forms at $2032 \mathrm{~cm}^{-1}$, while the peak at $2050 \mathrm{~cm}^{-1}$ is significantly diminished. Just prior to blistering, following an anneal to $339^{\circ} \mathrm{C}$, the peak at $2032 \mathrm{~cm}^{-1}$ shifts to $2027 \mathrm{~cm}^{-1}$ while a peak at $1969 \mathrm{~cm}^{-1}$ appears in the spectrum. At $399^{\circ} \mathrm{C}$, exfoliation has occurred and the $\mathrm{Ge}-\mathrm{H}$ modes in the spectrum originate from hydrogen residing below the cleavage plane and on free surfaces created by the blister process. In the spectrum taken with $s$-polarization in Fig. 7(a) there is still a 
TABLE I. Peak location as a function of temperature for peaks in the $1900-2100 \mathrm{~cm}^{-1}$ for $2 \times 10^{16}, 5 \times 10^{16}$ and $1 \times 10^{17} \mathrm{~cm}^{-2}$. When the peaks measured with $p$-polarization are shifted to other frequencies, these are indicated between parentheses. The peaks featured in MIT-FTIR spectra that show strong enhancement are indicated with an asterisk. Attribution of the measured peaks according to the literature reported values for discrete defect and extended surface modes of hydrogen in Ge.

\begin{tabular}{|c|c|c|c|c|}
\hline Temperature & $2 \times 10^{16}-$ Subcritical & $5 \times 10^{16}$ & $1 \times 10^{17}$ & Peak explanation \\
\hline \multirow[t]{4}{*}{ Unannealed } & 1763 & 1763 & 1763 & $1763 \mathrm{~cm}^{-1}$ : anti-bonding, stretch modes of $\mathrm{Ge}-\mathrm{H}_{2}^{*}$ at $10 \mathrm{~K}$ \\
\hline & 1979 & 1979 & 1979 & 1979: (100) Monohydride asymmetric stretch/ $\mathrm{VH}_{2}$ \\
\hline & 2008 & 2008 & 2008 & Hydrogenated Ge vacancy \\
\hline & 2050 & 2050 & $2050(2038)$ & $\mathrm{VH}_{4}$ or similar \\
\hline \multirow[t]{3}{*}{170} & & & 1996 & $\mathrm{VH}_{2}(1993)$ \\
\hline & 2008 & 2008 & 2008 & Hydrogenated Ge vacancy \\
\hline & 2050 & 2050 & $2050(2046)$ & $\mathrm{VH}_{4}$ \\
\hline \multirow[t]{3}{*}{221} & & 1996 & 1996 & $\mathrm{VH}_{2}(1993)$ \\
\hline & 2008 & 2008 & 2008 & Hydrogenated Ge vacancy \\
\hline & 2050 & 2050 & $2050(2046)$ & $\mathrm{VH}_{4}$ \\
\hline \multirow[t]{4}{*}{297} & & 1996 & 1996 & $\mathrm{VH}_{2}(1993)$ \\
\hline & 2008 & 2008 & 2008 & Hydrogenated Ge vacancy \\
\hline & $2040^{*}(2035)$ & $2030^{*}(2027)$ & $2033^{*}(2015)$ & $\mathrm{V}_{2} \mathrm{H}_{6}$ \\
\hline & 2050 & 2050 & 2050 & $\mathrm{VH}_{4} /$ trihydride \\
\hline \multirow[t]{4}{*}{339} & 1969 & 1969 & 1969 & 1969: (111) monohydride stretch \\
\hline & & 1996 & & 1996: (100) monohydride asymmetric stretch \\
\hline & 2008 & & 2008 & Hydrogenated Ge vacancy \\
\hline & $2038^{*}(2033)$ & $2029^{*}(2015)$ & $2027^{*}$ & $\mathrm{~V}_{2} \mathrm{H}_{6}$ \\
\hline \multirow[t]{4}{*}{399} & 1969 & 1969 & 1972 & 1969: (111) monohydride stretch \\
\hline & & 1990 & 1992 & 1996: (monohydride asymmetric stretch) \\
\hline & & 2001 & & Hydrogenated Ge vacancy/collective dihydride stretch \\
\hline & $2031^{*}(2033)$ & $2025^{*}(2008)$ & 2027 & $\mathrm{~V}_{2} \mathrm{H}_{6}$ \\
\hline \multirow[t]{4}{*}{501} & & $1962(1957)$ & & 1969: (111) monohydride stretch \\
\hline & & $1980(1970)$ & & 1980: (100) monohydride asymmetric stretch \\
\hline & & $1990(1986)$ & & 1990: (100) monohydride asymmetric stretch \\
\hline & $2008+(2021)$ & 2002 & & Hydrogenated Ge vacancy/collective dihydride stretch \\
\hline
\end{tabular}

strong contribution at $2027 \mathrm{~cm}^{-1}$. However, the contribution at $2008 \mathrm{~cm}^{-1}$ is no longer discernible, which agrees with the hypothesis that this mode corresponds to the hydrides present at the opening internal surfaces that contribute to the exfoliation process. A peak at $1991 \mathrm{~cm}^{-1}$ has formed, which is near the value of $1979 \mathrm{~cm}^{-1}$ reported by Chabal for the asymmetric stretch-mode of $\mathrm{Ge}(100)$ monohydride. ${ }^{10}$ This mode is polarized parallel to the surface and should therefore be observed with $s$-polarization for internal surfaces parallel to the surface, which should be observed in the -polarized spectra due to the orientation of the dipole for this mode.

The spectrum taken with $p$-polarization in Fig. 7(b) shows relatively little spectral detail due to the strong enhancement seen even prior to annealing. There is a slight shift of the dominant band at $2046 \mathrm{~cm}^{-1}$, relative to the dominant feature at $2050 \mathrm{~cm}^{-1}$ observed for samples implanted to $2 \times 10^{16}$ and $5 \times 10^{16} \mathrm{~cm}^{-2}$. Additionally, there is a broad shoulder toward lower frequencies that is suggestive of a peak near $2008 \mathrm{~cm}^{-1}$ as was observed in the spectrum taken with $s$-polarization for the unannealed sample. Annealing to $221{ }^{\circ} \mathrm{C}$ causes only a slight change in the peak profile along with the loss of the $\mathrm{Ge}-\mathrm{H}_{2}^{*}$ features at 1763 and $1979 \mathrm{~cm}^{-1}$. Upon annealing to $297{ }^{\circ} \mathrm{C}$, there is a dramatic increase in the enhancement indicating the onset of blistering accompanied by a shift of the dominant peak to $2031 \mathrm{~cm}^{-1}$. This dominant peak must be related to an internal structure with substantial $z$-component dipole. Such a H-decorated internal structure is, therefore, a precursor to the exfoliation of the thin film. Upon annealing to $339{ }^{\circ} \mathrm{C}$ there is further enhancement and sharpening of the major peak, which has now shifted down to $2008 \mathrm{~cm}^{-1}$. Finally, following a $399{ }^{\circ} \mathrm{C}$ annealing, the film is exfoliated and, as one would expect, the enhancement of the spectrum is no longer observed. In addition to the now diminished peak at $2008 \mathrm{~cm}^{-1}$, there are clearly distinguishable shoulders at 1992 and $2027 \mathrm{~cm}^{-1}$ that were likely present in the spectrum taken following the $339{ }^{\circ} \mathrm{C}$ anneal but remained unobserved due to the peak enhancement at $2008 \mathrm{~cm}^{-1}$ (Fig. 8). 


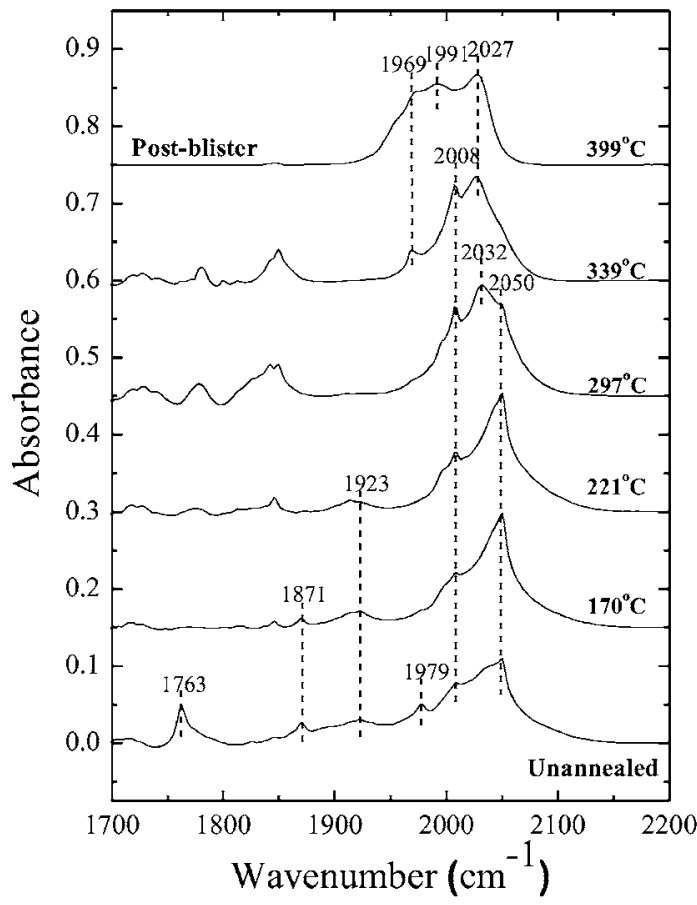

(a)

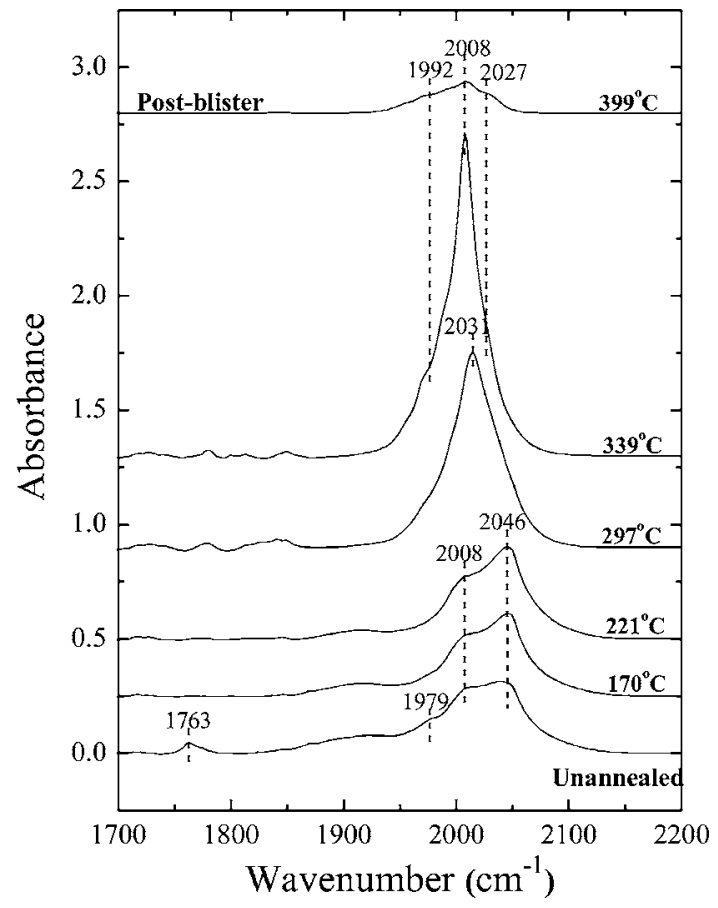

(b)

FIG. 7. (a) MIT-FTIR spectra taken with $s$-polarization for $1 \times 10^{16} \mathrm{~cm}^{-2}$ implanted Ge as a function of the isochronal annealing temperature. (b) MIT-FTIR spectra taken with $p$-polarization for $5 \times 10^{16} \mathrm{~cm}^{-2}$ implanted Ge as a function of the isochronal annealing temperature. The spectra for $399^{\circ} \mathrm{C}$ for both polarizations is taken following exfoliation of the implanted Ge layer.

\section{DISCUSSION}

In the preceding section, the essential elements of the exfoliation process in germanium were highlighted and discussed separately for the three different implantation doses. We now can combine these elements to first propose a coherent mechanism for the hydrogen induced exfoliation of germanium and then compare it with the mechanism developed elsewhere for silicon. ${ }^{7,23}$

The first important observation is that after implantation hydrogen is located mainly in defect structures such as hydrogenated vacancies with different degrees of hydrogenation. The dominant features in the spectrum taken with $s$-polarization are at 1979,2008 , and $2050 \mathrm{~cm}^{-1}$, which are respectively consistent with interstitial defects and monoand multi-hydrogenated vacancies. Following annealing, poorly hydrogenated defects tend to disappear in favor of more highly hydrogenated defects and internal planes. Interesting is the evolution of the peaks located at the highest frequencies, from 2008 to $2050 \mathrm{~cm}^{-1}$. The feature at $2050 \mathrm{~cm}^{-1}$ is attributed to the hydrogenated vacancy $\mathrm{VH}_{4}$, while the feature at $2027 \mathrm{~cm}^{-1}$ is attributed to agglomerated defect structures $\left(\mathrm{V}_{2} \mathrm{H}_{6}\right.$ and $\left.\mathrm{V}_{n} \mathrm{H}_{n+4}\right)$ that are precursors to blistering, as will be discussed below, and are still present in the substrate below the exfoliated region. The spectrum taken with $p$-polarization has a strongly enhanced peak at $2008 \mathrm{~cm}^{-1}$ that slightly remains following exfoliation only in the sample implanted with the highest dose. In the same sample and following exfoliation at $399^{\circ} \mathrm{C}$, there is a broad tail toward $1950 \mathrm{~cm}^{-1}$ that is consistent with the presence of Ge(111) surface monohydride modes. ${ }^{9}$ The mechanism de- scribed above is similar to the case of silicon, but there are some differences. In both cases there is a formation and transformation of hydrogenated vacancy species into extended internal surfaces. However, the nature of the vacancies contributing to the formation of the extended internal defects and the nature of hydrogen passivation inside the cavities are different. In the case of silicon it seems that $\mathrm{VH}_{2}$ vacancies are the main contributors to the exfoliation, while in the case of germanium the formation of larger vacancies (e.g., $\mathrm{V}_{2} \mathrm{H}_{6}$ and $\mathrm{V}_{n} \mathrm{H}_{n+4}$ ) appears to be the key metastable
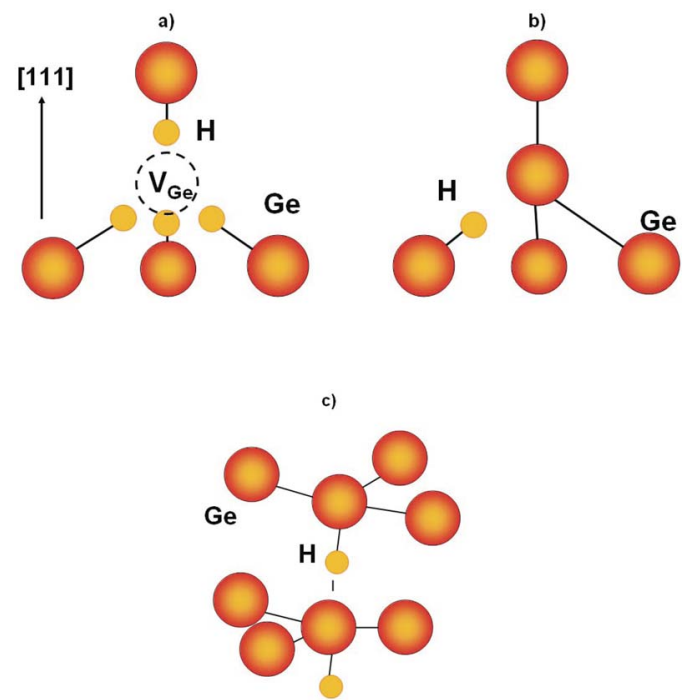

FIG. 8. (Color online) Basic schematics of the hydrogen in the (a) vacancy, (b) interstitial, and (c) $\mathrm{H}_{2}^{*}$ configurations. 
intermediate. Moreover, in silicon, cavities are passivated by monohydrides while in the case of germanium cavities seem to be passivated both by monohydrides and dihydrides. We believe that these results may explain why higher doses are needed for exfoliation of Ge compared to $\mathrm{Si}$ and point to subtle but important differences in these two otherwise similar materials.

\section{CONCLUSIONS}

Based upon the physical and chemical observations made by TEM, AFM, and MIT-FTIR, a qualitative understanding of the H-induced layer transfer process in Ge has been achieved. Similarly to the case of $\mathrm{Si}$, defects are formed in the germanium structure upon H-implantation, which trap hydrogen in a large variety of modes assigned by FTIR spectroscopy to $\mathrm{Ge}-\mathrm{H}_{2}^{*}, \mathrm{~V}_{2} \mathrm{H}_{6}$, and $\mathrm{V}_{n} \mathrm{H}_{n+4}$. Upon annealing, some of these structures are lost and the hydrogen therein is free to move through diffusion to more stable configurations such as internal (100) and (111) surfaces, which act as the nucleation points for internal cavities and platelets. This is further supported by XTEM observation of (100)-oriented defect structures typical of platelets immediately following implantation and by the possible spectroscopic signal at $1996 \mathrm{~cm}^{-1}$ assigned to (100) platelet structures. Upon sufficient annealing micro-cracks form along the same orientation in the crystal, lying predominantly along the (100) plane, as observed by XTEM. Simultaneously, vibrational frequencies associated with $\mathrm{Ge}(100)$ surface mono- and dihydride appear in the FTIR spectra, indicating that hydrogen provides a termination species for the internal blister structures. These micro-cracks and their agglomerated precursors serve as points for the coalescence of $\mathrm{H}_{2}$ and the development of internal pressure sufficient to break bonds and extend the internal fracture laterally. Upon ripening of the micro-cracks into supercritical radius internal surfaces, blisters pop-up and lead to the exfoliation of the thin Ge film. This work, therefore, confirms that the exfoliation process in $\mathrm{Ge}$ is similar to that in $\mathrm{Si}$, despite differences in $\mathrm{Ge}-\mathrm{H}$ bond strength and $\mathrm{Ge}$ chemical activity.

\section{ACKNOWLEDGMENT}

The authors kindly acknowledge Martin M. Frank for helpful discussions and insights in the MIT experiments.
${ }^{1}$ M. Bruel, Electron. Lett. 31, 1201 (1995).

${ }^{2}$ U. Goesele and Q. Tong, Annu. Rev. Mater. Sci. 28, 215 (1998).

${ }^{3}$ J. Zahler, C. Ahn, S. Zaghi, H. A. Atwater, C. Chu, and P. Iles, Thin Solid Films 403, 558 (2002).

${ }^{4}$ A. Fontcuberta i Morral, J. Zahler, and H. A. Atwater, Appl. Phys. Lett. 83, 5413 (2003).

${ }^{5}$ I. Radu, I. Szafraniak, R. Scholz, M. Alexe, and U. Goesele, J. Appl. Phys. 94, 7820 (2003).

${ }^{6}$ S. Hayashi, D. Bruno, and M. Goorsky, Appl. Phys. Lett. 85, 236 (2004).

${ }^{7}$ M. Weldon, V. Marsico, Y. Chabal, A. Agarwal, D. Eaglesham, J. Sapjeta, W. Brown, D. Jacobson, Y. Caudano, S. Christman et al., J. Vac. Sci. Technol. B 15, 1065 (1997).

${ }^{8}$ M. Weldon, M. Collot, Y. Chabal, V. Venezia, A. Agarwal, T. Haynes, D. Eaglesham, S. Christman, and E. Chaban, Appl. Phys. Lett. 73, 3721 (1998).

${ }^{9}$ A. Fontcuberta i Morral, J. Zahler, M. Griggs, H. A. Atwater, and Y. Chabal, Phys. Rev. B 72, 085219 (2005).

${ }^{10}$ S. Myers, H. Stein, and D. Follstaedt, Phys. Rev. B 51, 9742 (1995).

${ }^{11}$ S. Myers, D. Follstaedt, G. Petersen, C. Seager, H. Stein, and W. Wampler, Nucl. Instrum. Methods Phys. Res. B 106, 379 (1995).

${ }^{12}$ Y. Chabal, Surf. Sci. Rep. 8, 211 (1988).

${ }^{13}$ M. Weldon, Y. Chabal, S. Christman, E. Chaban, L. Feldman, and D. Hamann, J. Vac. Sci. Technol. B 14(4), 3095 (1996).

${ }^{14}$ M. Budde, B. Nielsen, P. Leary, J. Goss, R. Jones, P. Briddon, S. Oberg, and S. Breuer, Phys. Rev. B 57, 4397 (1998).

${ }^{15}$ M. Budde, B. Nielsen, R. Jones, J. Goss, and S. Oberg, Phys. Rev.
B 54, 5485 (1996).

${ }^{16}$ D. Feijoo, Y. Chabal, and S. Christman, Appl. Phys. Lett. 65, 2548 (1994).

${ }^{17}$ Y. Chabal, M. Hines, and D. Feijoo, J. Vac. Sci. Technol. A 13, 1719 (1995).

${ }^{18}$ Following implantation visual and spectroscopic evidence indicated the presence of surface oxidation due to increased base pressure near the sample in the gas cooled implantation stage. Following implantation, there is a large amount of absorption in the region where one would expect the $\mathrm{O}-\mathrm{H}$ stretch of water to be observed. Additionally, absorption in the Ge-O region near $1000 \mathrm{~cm}$ ? further corroborates the presence of a water saturated oxide. By etching the sample in 10 p.c. HF for approximately $30 \mathrm{~min}$, this contamination was removed and a subsequent spectrum shows little or no absorption in these regions. The contribution of contamination due to annealing was determined by the observation of peak growth in both the water and germanium oxide modes upon annealing.

${ }^{19}$ S. Myers, H. Stein, and D. Follstaedt, Phys. Rev. B 51, 9742 (1995).

${ }^{20}$ Y. Chabal, Surf. Sci. 168, 594 (1986).

${ }^{21}$ F. Maroun, F. Ozanam, and J. Chazalviel, Chem. Phys. Lett. 292, 493 (1999)

${ }^{22}$ S. Rivillon, Y. Chabal, F. Amy, and A. Kahn, Appl. Phys. Lett. 87, 253101 (2005).

${ }^{23}$ M. Weldon, M. Collot, Y. Chabal, V. Venezia, A. Agarwal, T. Haynes, D. Eaglesham, S. Christman, and E. Chaban, Appl. Phys. Lett. 73, 3721 (1998). 